\title{
Beyond Val30Met transthyretin (TTR): variants associated with age-at-onset in hereditary ATTRv amyloidosis
}

\section{Miguel Alves-Ferreiraa,b, Ana Azevedob, Teresa Coelhoc, Diana Santos ${ }^{a, b}$, Jorge Sequeiros ${ }^{a, b}$, Isabel Alonso ${ }^{a}$, Alda Sousa ${ }^{a, b}$ and Carolina Lemos ${ }^{a, b *}$}

${ }^{a}$ UnIGENe, IBMC - Institute for Molecular and Cell Biology, i3S - Instituto de Investigação e Inovação em Saúde, Universidade do Porto, Porto, Portugal; ${ }^{b}$ ICBAS - Instituto Ciências Biomédicas Abel Salazar, Universidade do Porto, Portugal; ' Unidade Corino de Andrade (UCA), Centro Hospitalar do Porto (CHP), Porto, Portugal

*Corresponding author:

Carolina Lemos, PhD

Invited Auxiliary Professor, ICBAS

UnIGENe, IBMC-i3S

Rua Jorge Viterbo Ferreira, 228

4050-313 Porto

Telephone: +351220428002

E-mail: clclemos@ibmc.up.pt

This is an Accepted Manuscript of an article published by Taylor \& Francis in Amyloid on 18 Jan 2021, available online: https://www.tandfonline.com/doi/full/10.1080/13506129.2020.1857236 


\section{ABSTRACT}

Objectives: ValzoMet in transthyretin (TTR) gene is causative for familial amyloid polyneuropathy (FAP). FAP shows a wide variation in age-at-onset (AO) between clusters, families, and among generations. We aim at identifying genetic modifiers of disease onset that may contribute to this variability in Portuguese patients by identifying other variants in TTR locus, beyond the TTR-FAP causing variant that could play a regulatory role in its expression level.

Methods: We analysed DNA samples of 330 Val3oMet carriers (299 patients, 31 aged-asymptomatic carriers aged $>40$ years) from 120 families currently under follow-up. A generalized estimating equation analysis (GEE) was used to take into account non-independency of $A O$ between relatives. An intensive in silico analysis was performed in order to understand a possible regulation of gene expression.

Results: We found 11 rare variants in the promoter, coding and intron/exon boundaries of the TTR gene associated with the onset of symptoms before and after age 40 years, namely 2 novel ones and a tandem CA-dinucleotide repeat. The seven ValzoMet/ValzoMet homozygous do not carry any of the variants identified in this study, including the common ones. In silico analysis disclosed significant alterations in the mechanism of splicing, transcription factors and miRNAs binding.

Conclusions: Variants within the promoter region may modify disease expressivity and variants in the 3'UTR can impact the efficacy of novel therapeutic interventions. Importantly, the putative mechanisms of regulation of gene expression within the TTR gene deserve to be better explored, in order to be used in the future as potential therapeutical targets.

Keywords: Age-at-onset, familial amyloid polyneuropathy, TTR gene, promoter, Hereditary transthyretin, genetic modifiers 


\section{INTRODUCTION}

Transthyretin-related familial amyloid polyneuropathy (TTR-FAP) is an autosomal dominant neurodegenerative disease that presents as a progressive sensorimotor and autonomic polyneuropathy with involvement of the small myelinated and unmyelinated nerve fibers $[\underline{1}, \underline{2}]$. It is characterized by mutated protein deposition in the form of amyloid substance (ATTRm). Without treatment, it leads to death within 10-20 years [1]. The Val3oMet variant (p.Val5oMet) in the transthyretin gene (TTR) is the commonest causative variant for TTR-FAP [3]. Significant phenotypic heterogeneity, namely in age-at-onset (AO), has been described in ValzoMet patients. Variation between clusters has been widely documented, with Portuguese, Brazilian and Japanese patients showing mostly an early-onset $(\mathrm{AO}<40)$, whereas Swedish patients are known for their late-onset $(\geq 50)[4-6]$. However, within each cluster variation is equally large, and range in AO quite similar. In Portuguese patients clinical symptoms typically occur before age 40 years (early-onset), with lateonset patients being increasingly ascertained, leading to a wide variation in AO (19-82 years) [ $\mathrm{z}]$. The most intriguing feature is variation within the same family and the fact that late-onset patients often have very early-onset offspring, while the reverse was never observed [ $]$ ].

In recent years, we explored the role of common variants in different candidate genes involved in different TTR pathways, taking into account the family structure and the non-independency of AO among members of the same family [8-10]. Although these results bring new insights on TTR pathways, AO variation is not fully explained. The observed intra-generational variability; the fact that late-onset patients have early-onset offspring and due to the main role of TTR in FAP, this led us to focus in another approach to identify modifiers closely linked to the TTR locus.

A small part of this variability may be explained by non-coding genetic variants in cis[11] and a transacting factor[12], but additional factors clearly remain to be discovered.

We speculated that variants within the regulatory regions of the TTR gene might account for the changed gene expression and ultimately culminate in phenotypic heterogeneity. The aim of this study was then to identify other variants and regulatory factors at TTR locus and understand how they may contribute as genetic modifiers of AO in TTR-FAP. 


\section{Subjects and Methods}

\section{Subjects and families}

Unidade Corino de Andrade - Centro Hospitalar Universitário do Porto (UCA-CHUP, Porto) has the largest TTR-FAP ValzoMet registry worldwide (more than 3000 patients), collected over 80 years and clinically well characterized. We selected 120 families currently under follow-up, with at least 2 generations affected and coming from all geographical areas of Portugal. Our study sample consists of 330 ValzoMet carriers (299 patients, 5 homozygous; 31 asymptomatic carriers, 2 homozygous).

For each patient, AO was defined by the same team of neurologists specialized in Val3oMet TTR-FAP, considering the presence of a whole set of symptoms characteristic of small fibers' neuropathy and not isolated and unspecific symptoms [10]. As for AO, it has long been accepted to classify patients in three groups: early ( $\leq 40)$, intermediate (40-49) and late ( $\geq 50)$ [13]. In this study, patients were divided in two groups only: early $(\leq 40)$ and intermediate/late-onset (>40 years). All asymptomatic carriers were aged 40 years or more and therefore were also included in the intermediate/late-onset group. The presence of ValzoMet variant was confirmed in all subjects. DNA samples were collected at UCA-CHUP and stored at Centro de Genética Preditiva e Preventiva (CGPP-IBMC, Porto) biobank, authorized by CNPD (National Commission for Data Protection). The study was approved by the Ethics Committee of CHUP. Written informed consent was obtained from all patients.

\section{Genotyping}

Genomic DNA was extracted either from peripheral blood leucocytes or saliva, using the QIAamp ${ }^{\circledR}$ DNA Blood Mini Kit (QIAGEN ${ }^{\mathrm{TM}}$ ) and Oragene DNA Self Collection Kits (DNA Genotek, Ottowa, Canada) according to manufacturer's instructions, respectively.

The promoter, coding and all intron/exon boundaries of the TTR gene were amplified by polymerase chain reaction (PCR) using HotStarTaq DNA Polymerase ${ }^{\circledR}\left(\right.$ Qiagen $\left.^{\mathrm{TM}}\right)$ and primers forward and reverse for each amplicon (sequences available upon request).

Then the PCR products were examined by direct Sanger sequencing using dye terminator chemistry approach from Applied Biosystems ${ }^{\mathrm{TM}}$, with the same set of primers as for amplification, and samples loaded on an ABI-PRISM 3130 XL genetic analyzer (Applied Biosystems).

\section{Statistical analysis}

Association of genetic variants with $\mathrm{AO}$ (as a dichotomous variable), as in the case of the tandem repeat 
and the two variants together, was explored using Chi-Square or Fisher's exact test. AO was also analyzed as a continuous variable: to account for non-independency of AO between members of the same family, we used generalized estimating equations (GEE); to correct for multiple testing, we applied a Bonferroni correction, dividing by the number of GEE tests performed. Therefore, statistical significance was set at $\alpha<0.0125$. All statistical analyses were performed using IBM SPSS Statistics software (v.24).

\section{In silico analysis}

To predict the impact of sequence variants on TTR function, bioinformatics tools included in the Alamut Mutation Interpretation Software (Interactive Biosoftware, Rouen France) and Polyphen-2 were applied.

Also, we ran a comprehensive bioinformatics package, FuncPred[14], organized by $\mathrm{NIH}$ and one of the most reliable and popular function-prediction tools.

In order to predict potential consequences of variants on splicing events, we used two prediction programs (ESEfinder and Human Splicing Finder) in Alamut Software.

The JASPAR[15] database was utilized to explore the transcription factors binding (TFB) capacity. Transcription factors were filtered by expression in liver with an expression level greater than 1 Transcripts per Million (TPM) using Genotype-Tissue Expression (GTEx) project.

miRNA target sites in the $3^{\prime} U T R$ TTR-wild type (WT) were predicted by miRWalk[16] and we used PITA algorithm, taking in account the conservation and the differences in the alignment scores [17]. 


\section{Results}

\section{Study group demographic characteristics}

This study included 330 ValzoMet carriers (174 females, 156 males). Among the 299 patients, AO ranged from 21-74 years (mean 38.7; SD 12.9). The group with $A O \leq 40$ had 202 patients, whereas the group with $\mathrm{AO}>40$ consisted of 97 patients plus 31 asymptomatic carriers (aged 42 to 90 years) who might be considered similar to intermediate/late-onset patients.

Interestingly, of the 330 carriers, 7 were homozygous: 3 had onset >50, 2 were asymptomatic at age 50 or more and 2 had an onset $<50$.

We sequenced $2.1 \mathrm{~kb}$ upstream TTR to include promoter region and the coding and flanking regions of the TTR gene. Location and frequency of the variants found are shown in Table 1 [near here].

\section{Common variants}

As shown in Table 1, we identified four common variants (MAF >10\%), 3 in the promoter region and 1 in $3^{\prime}$ near gene. TT genotypic of rs1791228 variant revealed on GEE analysis an association with AO $(p<0.0125)(B=23,6 ; 95 \% C l, 20,2$ to 27,0$)$, significant after multiple-testing correction.

Interestingly one variant is a tandem repeat composed by a CA-dinucleotide (rs71383038) in the promoter region (Table 1). While the most common allele in the Portuguese population (including our sample) was $C A 10$, followed by the minor allele CA9, we found an unreported allele with seven CArepeats ( $C A 7$ ). When we compared $C A 9$ carriers with $C A 10$, the only two $C A 9$ homozygotes for the tandem repeat rs 71383038 showed onset after 60 years; $56.1 \%$ of the patients presented the common genotype ( $\left.\mathrm{CA}_{10} / \mathrm{CA}_{10}\right)$ and $42.7 \%$ the $\mathrm{CAg} / \mathrm{CA}_{10}$ genotype. Worth mentioning, the only patient with a deletion of 3 CA-repeats (CA7) showed a very early-onset (30 years).

None of the other common variants presented significant differences in frequencies when comparing early- and intermediate/late-onset patients or when analyzing $\mathrm{AO}$ as a continuous variable. The variants rs3764479 and rs3794885 were the most common alterations in the promoter region ( $43.5 \%$ and $42.8 \%$, respectively).

Noteworthy, in the $\mathrm{AO} \leq 40$ group, more patients carried simultaneously the minor allele of rs3764479 and of $r s 3764478$ ( $p=0.03)$, with a 2.22 -fold risk for symptoms to start before age $40(O R=2.22,95 \%$ $\mathrm{Cl}=1.05$ to 4.70$)$. 


\section{Rare Variants}

In the promoter region, 12 rare variants were found, including one novel (c. -1993 G>T). Seven rare variants in the coding and flanking regions of the TTR gene were identified, including two novel ones: C.105A $>$ G, in exon 3 (p.Lys35Lys), a synonymous variant and C.200+107 T>C, in intron 2. Variants already reported included 2 intronic, two at the $3^{\prime}$ UTR and 2 exonic ones. Importantly, rare variants: C. -1993 G>T; rs13381522; rs546878244; rs3764477; rs540872876; rs58616646; 146750662 and rs996949357 in the promoter region and C.200+107 T>C; rs28933981; and rs1053907197 in the coding and flaking regions of TTR gene showed an association with $A O(p<0.0125)$, significant after multipletesting correction (Table 2 near here).

Regarding the coding variant, TTR Ser6 (rs1800458, p.Gly26Ser), ten patients showed an early-onset and eight patients a late-onset, ( $p>0.05)$, showing that TTR Ser6 is not associated with early or lateonset. All compound heterozygotes for TTR ValzoMet/Thr119Met ( $p$.Val50Met/Thr139Met) showed a later-onset, as previously described [18].

\section{Homozygous individuals}

ValzoMet/ValzoMet homozygous do not carry any of the variants identified in this study: they are also homozygous for the common allele of each variant.

\section{Haplotype Analysis}

We performed a haplotype analysis (using the Haploview software) with the variants identified in this study and we did not find any significant results (Table 3 near here).

\section{Putative effects of the rare genetic variants}

Using JASPAR database and GTEx project, we predicted that the combination of rs3764479/rs3764478 variants (as they were found to be present mostly together) create 69 new potential transcriptionfactor binding sites and may disrupt another 18, all expressed in the liver, where TTR is synthetized (Table 4 near here).

We also assess how likely a splicing change would occur as a result of the presence of DNA variants. Table 5 [near here] shows the variants that may alter splicing significantly (consensus value (CV) higher than 70). We found a new acceptor site, 2 nucleotides downstream rs 1800458 , probably an active splice site. A branch point, probably inactive in the wild-type sequence, is predicted to become a strongly active splice site in the presence of rs1791228. Also, presence of $r 536204272$ leads to loss of a donor site 
probably inactive in wild-type TTR. Disruption of four exonic splicing enhancers was observed in rs28933981, while creation of three new ones was seen in rs1800458.

Using miRNA-target prediction programs, we detected two miRNAs: mir-200a and mir-141, which are predicted target-sites for the TTR 3'UTR. Presence of rs62093482 or rs1053907197 (located in TTR $3^{\prime}$ UTR) does not interfere with miRNA binding. rs62093482 creates a putative target site to mir-622. The rare allele of rs1053907197 is a putative target site to mir-138 and mir-622. 


\section{Discussion}

TTR-FAP phenotypic variability is evident not only between different disease-associated variants but also for patients with the same disease-causing variant that display different clinical manifestations. The main focus of this study was on the phenotypic variability of the TTR-FAP ValzoMet individuals regarding $\mathrm{AO}$.

\section{Phenotypic effect of variants}

Using a parametric test (GEE), which is a quite powerful statistical method, we found interesting results regarding the association of some of these variants with $\mathrm{AO}$, which showed that rare variants in fact seem to modulate AO variability. The effect size of the variants found could be small, which can be a limitation in order to achieve a significant association with AO, due to statistical power. Likewise, how to point out rare variants as a causative effect on $\mathrm{AO}$ remains challenging, as they may not be shared by all affected individuals. Although the role of environmental factors cannot be excluded, even as, the contribution of other genetic factors (common or rare) and a global role of them in modulation of specific phenotypes in the presence of the same disease causing variant.

We found significant results for some rare variants: despite the fact that the heterozygous genotype appeared only once, this is in accordance with the allelic frequencies described for European (NonFinnish) in Genome Aggregation Database (GnomAD). Moreover, we could not preclude the possibility that high effect risk alleles were present. Thus, we consider that these rare variants results are equally important and should be reported, because their identification may have implications for genetic screening and personalized treatment.

The protective role of $\mathrm{rs} 1800458$ (p.Gly26Ser) has already been hypothesized[11,19]; however, results do not allow us to state that this coding variant was protective in our Val3oMet patients, since it did not significantly associate with late-onset.

Both Thr11gMet carriers found had a very late $\mathrm{AO}$ ( 61 and 63 years), though they belonged to an earlyonset family. This supports the theory that stability of the TTR molecule may be an important factor to prevent amyloidogenesis, offering a protection from the Val3oMet effects $[18,20]$.

As described in other clusters [21,22], the homozygous status does not appear to be related to the onset of symptoms, as we found late-onset carriers of ValzoMet/Val3oMet. Interestingly, the 7 homozygous analyzed in this study do not carry any of variants identified. This leads us to hypothesize that the absence of these variants in the TTR vicinity may compensate the double genetic dose of TTR 
Val3oMet.

Noteworthy, it is very promising that the combination of the minor alleles of rs3764479 and rs3764478 (which appear frequently together) showed a high risk for an early-AO. This approach deserves our future attention, as clusters of patient-specific variant combinations have already been found [23].

\section{Putative effect on splicing activity, TFBSs and miRNA target sites}

Analysis of non-coding regions suggests the presence of four non-coding variants, possibly cisregulatory elements of the TTR gene that modulate the effect of ValzoMet on the disease phenotype.

To the best of our knowledge, no other studies about genetic alterations in splicing activity on TTR gene have been performed, except for a recent association study for variants in the TTR gene in Han Chinese patients with Alzheimer disease [24]. It is important to emphasize that some of the differences between WT and variant sequences could alter splicing either by re-directing the spliceosome or by altering the binding of auxiliary factors, such as SR proteins, exonic and intronic splicing enhancers and silencers [25]. For instance, results showed that variant rs1791228 was significantly associated with AO, and the association survived Bonferroni correction. According to the splicing events' prediction programs, this variant may influence the constitutive splicing of TTR pre-mRNA.

The TTR gene is regulated by the promoter at the transcriptional level in liver cells [26]. In this study, we identified two variants in the promoter region that together reduce significantly AO. In silico, these variants not only disrupt potential binding sites once occupied by transcription factors, but also create new ones. Human transcription factors were predicted with a relative profile score threshold $>80 \%$ and filtered by expression in liver.

miRNA mir-200a and mir-141 inhibit TTR expression, by directly binding to the 3 'UTR of TTR, which is reversed by variants in the miRNA binding site [27]. rs62093482 and rs1053907197 variants do not affect the $3^{\prime}$ UTR miRNA binding sites, but they create new ones. Two novel therapeutic drugs recently approved for treatment of FAP modulate TTR expression at the RNA level: patisiran, the first small interfering RNA-based drug approved by the FDA [28]; and inotersen, an antisense oligonucleotide [29]. Since both drugs prevent the production of TTR by targeting its $3^{\prime}$ UTR, we hypothesized that variants in this region could modify their efficacy.

As we are aware, this is the largest screening study of TTR variants in ValzoMet TTR-FAP. Our data suggest possible mechanisms that may account for expression of TTR as a potential modulator of the course of FAP. This reinforces the idea that molecular diagnosis should go beyond simply screening for the disease-causing variant. 


\section{Acknowledgments}

We thank all the patients and families for participating in this study and Joana Silva for all the help with the optimization of protocol for sequencing. We would like to thank FEDER funds, through the Programa Operacional Factores de Competitividade - COMPETE 2020 and by Nacional funds through the FCT - Fundação para a Ciência e a Tecnologia [COMPETE: POCl-01-0145-FEDER-007440]. This work was supported by grants of Fundação para a Ciência e Tecnologia, FCT [PTDC/SAUGMG/100240/2008 and PEsT], co-funded by ERDF and COMPETE; and by Financiamento Plurianual de Unidades de Investigação (FCT). MAF is recipient of a FCT fellowship [SFRH/BD/101352/2014]. The funders had no role in the design and conduct of the study; collection, management, analysis, or interpretation of the data; preparation, review, or approval of the manuscript; or decision to submit the manuscript for publication.

Disclosure of interest: T.C.'s institution has received support from FoldRx Pharmaceuticals, which was acquired by Pfizer Inc in October 2010; T.C. has served on the scientific advisory board of Pfizer Inc and received funding from Pfizer Inc for scientific meeting expenses (travel, accommodations, and registration). T.C. currently serves on the THAOS (natural history disease registry) scientific advisory board.

The other authors have no conflicts of interest. 


\section{Reference}

1.

2.

Andrade C. A peculiar form of peripheral neuropathy; familiar atypical generalized amyloidosis with special involvement of the peripheral nerves. Brain. 1952 Sep;75(3):408-27.

Said G, Ropert A, Faux N. Length-dependent degeneration of fibers in Portuguese amyloid polyneuropathy: a clinicopathologic study. Neurology. 1984 Aug;34(8):1025-32.

Saraiva MJ, Birken S, Costa PP, et al. Amyloid fibril protein in familial amyloidotic polyneuropathy, Portuguese type. Definition of molecular abnormality in transthyretin (prealbumin). J Clin Invest. 1984 Jul;74(1):104-19.

Rapezzi C, Quarta CC, Riva L, et al. Transthyretin-related amyloidoses and the heart: a clinical overview. Nat Rev Cardiol. $2010 \mathrm{Jul} ; 7(7): 398-408$.

Waddington-Cruz M, Schmidt $\mathrm{H}$, Botteman MF, et al. Epidemiological and clinical characteristics of symptomatic hereditary transthyretin amyloid polyneuropathy: a global case series. Orphanet J Rare Dis. 2019 Feb 8;14(1):34.

Sekijima $Y$, Ueda M, Koike $H$, et al. Diagnosis and management of transthyretin familial amyloid polyneuropathy in Japan: red-flag symptom clusters and treatment algorithm. Orphanet J Rare Dis. 2018 Jan 17;13(1):6.

Lemos C, Coelho T, Alves-Ferreira M, et al. Overcoming artefact: anticipation in 284 Portuguese kindreds with familial amyloid polyneuropathy (FAP) ATTRV $30 M$. J Neurol Neurosurg Psychiatry. 2014 Mar;85(3):326-30.

Santos D, Coelho T, Alves-Ferreira M, et al. Variants in $\mathrm{RBP}_{4}$ and AR genes modulate age at onset in familial amyloid polyneuropathy (FAP ATTRV $30 M$ ). Eur J Hum Genet. 2016 May;24(5):756-60.

Santos D, Coelho T, Alves-Ferreira M, et al. Familial amyloid polyneuropathy in Portugal: New genes modulating age-at-onset. Ann Clin Transl Neurol. 2017 Feb;4(2):98-105.

Dias A, Santos D, Coelho T, et al. $C_{1} \mathrm{QA}$ and $\mathrm{C}_{1} \mathrm{OC}$ modify age-at-onset in familial amyloid polyneuropathy patients. Ann Clin Transl Neurol 2019.

Sikora JL, Logue MW, Chan GG, et al. Genetic variation of the transthyretin gene in wildtype transthyretin amyloidosis (ATTRwt). Hum Genet. 2015 Jan;134(1):111-21.

Alves-Ferreira M, Coelho T, Santos D, et al. A Trans-acting Factor May Modify Age at Onset in Familial Amyloid Polyneuropathy ATTRV30M in Portugal. Mol Neurobiol. 2017 May 19.

Coelho T, Sousa A, Lourenco E, et al. A study of 159 Portuguese patients with familial amyloidotic polyneuropathy (FAP) whose parents were both unaffected. J Med Genet. 1994 Apri31(4):293-9.

Xu Z, Taylor JA. SNPinfo: integrating GWAS and candidate gene information into functional SNP selection for genetic association studies. Nucleic Acids Res. 2009 Jul;37(Web Server issue):W600-5. 
15. Khan A, Fornes O, Stigliani A, et al. JASPAR 2018: update of the open-access database of transcription factor binding profiles and its web framework. Nucleic Acids Res. 2018 Jan 4;46(D1):D260-D266.

16. Dweep H, Gretz N. miRWalk2.o: a comprehensive atlas of microRNA-target interactions. Nat Methods. 2015 Aug;12(8):697.

17. Kertesz $M$, lovino $N$, Unnerstall $U$, et al. The role of site accessibility in microRNA target recognition. Nat Genet. 2007 Oct;39(10):1278-84.

18. Coelho T, Chorão R, Sousa A, et al. Compound heterozygotes of transthyretin Metzo and transthyretin Met119 are protected from the devastating effects of familial amyloid polyneuropathy. Neuromuscular Disorders. 1996;6( Supplement 1):S20.

19. Fitch NJ, Akbari MT, Ramsden DB. An inherited non-amyloidogenic transthyretin variant, [Ser6]-TTR, with increased thyroxine-binding affinity, characterized by DNA sequencing. J Endocrinol. 1991 May;129(2):309-13.

20. Hammarstrom $P$, Wiseman RL, Powers ET, et al. Prevention of transthyretin amyloid disease by changing protein misfolding energetics. Science. 2003 Jan 31;299(5607):713-6.

21. Holmgren $\mathrm{G}$, Hellman $\mathrm{U}$, Lundgren $\mathrm{HE}$, et al. Impact of homozygosity for an amyloidogenic transthyretin mutation on phenotype and long term outcome. J Med Genet. 2005 Dec;42(12):953-6.

22. Yoshinaga T, Nakazato M, Ikeda S, et al. Homozygosity for the transthyretin-Metzo gene in three Japanese siblings with type I familial amyloidotic polyneuropathy. Neurology. 1992

Oct;42(10):2045-7.

23. Mellerup E, Moller GL. Combinations of Genetic Variants Occurring Exclusively in Patients. Comput Struct Biotechnol J. 2017;15:286-289.

24. Xiang $\mathrm{Q}, \mathrm{Bi} \mathrm{R}, \mathrm{Xu} \mathrm{M}$, et al. Rare Genetic Variants of the Transthyretin Gene Are Associated with Alzheimer's Disease in Han Chinese. Mol Neurobiol. 2017 Sep;54(7):5192-5200.

25. Cartegni L, Chew SL, Krainer AR. Listening to silence and understanding nonsense: exonic mutations that affect splicing. Nat Rev Genet. 2002 Apr;3(4):285-98.

26. Wang Z, Burke PA. Hepatocyte nuclear factor-4alpha interacts with other hepatocyte nuclear factors in regulating transthyretin gene expression. FEBS J. 2010 Oct;277(19):406675 .

27. Saha S, Chakraborty S, Bhattacharya A, et al. MicroRNA regulation of Transthyretin in trophoblast differentiation and Intra-Uterine Growth Restriction. Scientific Reports. $2017 ; 7(1)$.

28. Alnylam PI. Onpattro (patisiran) lipid complex injection, for intravenous use: US prescribing information. : http://www.fda.gov/, ; 2018; Accessed 4 Out 2018.:[

29. EU. EMA-E. Tegsedi International non-proprietary name: inotersen. Assessment report. 2018 [cited 31 May 2018]. 
Table 1: Variants found in the TTR locus: dbSNP ID, location, frequency in AO $\leq 40$ and $>40$ onset patients, and minor allele frequency in Val3oMet Portuguese patients and in the Genome Aggregation Database (GnomAD).

\begin{tabular}{|c|c|c|c|c|c|c|c|c|}
\hline \multirow{2}{*}{\multicolumn{2}{|c|}{ TTR Region }} & \multirow[b]{2}{*}{ dbSNP ID } & \multirow{2}{*}{$\begin{array}{c}\text { Location } \\
\text { NM_000371.3 (HGVS) }\end{array}$} & \multirow[b]{2}{*}{$\mathbf{N}$} & \multicolumn{2}{|c|}{ Variant frequency } & \multicolumn{2}{|c|}{ Allele Frequency } \\
\hline & & & & & $A O \leq 40$ & $A O>40$ & Val30Met & $\begin{array}{c}\text { European } \\
\text { (Non-Finish) }\end{array}$ \\
\hline \multirow{15}{*}{\multicolumn{2}{|c|}{ 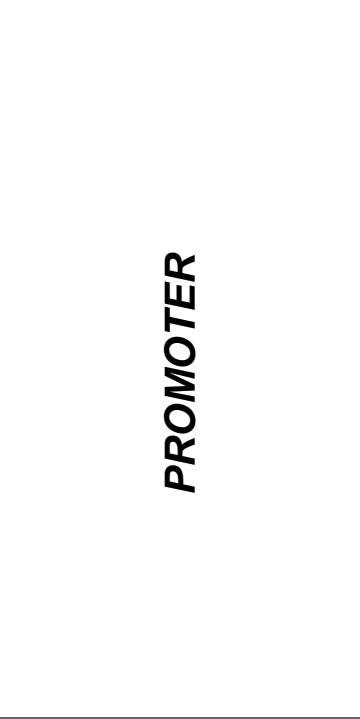 }} & rs3764479 & c. $-2041 A>G$ & 111 & 64 & 47 & 0.222 & 0.328 \\
\hline & & & c. $-1993 \mathrm{G}>\mathrm{T}$ & 1 & 1 & 0 & 0.002 & - \\
\hline & & rs13381522 & c. $-1933 \mathrm{C}>\mathrm{T}$ & 7 & 3 & 4 & 0.014 & 0.046 \\
\hline & & rs546878244 & c. $-1700 \mathrm{G}>\mathrm{A}$ & 1 & 1 & 0 & 0.002 & 0.000 \\
\hline & & rs3764478 & c. $-1383 G>T$ & 39 & 27 & 12 & 0.078 & 0.111 \\
\hline & & rs71383038 & c. $-1232 \_-1231$ delCA & 112 & 64 & 48 & 0.220 & - \\
\hline & & rs72922940 & c. $-1168 \bar{A}>G$ & 35 & 18 & 17 & 0.069 & 0.116 \\
\hline & & rs3764477 & c. $-1157 \mathrm{G}>\mathrm{A}$ & 9 & 4 & 5 & 0.018 & 0.045 \\
\hline & & rs540872876 & c. $-1156 \mathrm{G}>\mathrm{A}$ & 1 & 0 & 1 & 0.002 & 0.000 \\
\hline & & rs58616646 & c. $-1136 \mathrm{C}>\mathrm{T}$ & 8 & 4 & 4 & 0.016 & 0.046 \\
\hline & & rs146750662 & c. $-950 \mathrm{C}>\mathrm{T}$ & 2 & 2 & 0 & 0.004 & 0.004 \\
\hline & & rs116409170 & c. $-833 C>T$ & 6 & 3 & 3 & 0.012 & 0.025 \\
\hline & & rs3794885 & c. $-743 \mathrm{~A}>\mathrm{T}$ & 109 & 63 & 46 & 0.218 & 0.296 \\
\hline & & rs79748512 & c. $-682 G>A$ & 6 & 2 & 4 & 0.012 & 0.039 \\
\hline & & rs996949357 & c. $-543 T>C$ & 1 & 0 & 1 & 0.002 & - \\
\hline \multirow{8}{*}{ 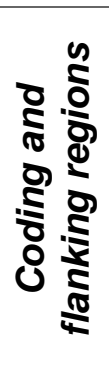 } & $\ln 2$ & & c. $200+107 \mathrm{~T}>\mathrm{C}$ & 1 & 0 & 1 & 0.002 & - \\
\hline & Ex 2 & rs 1800458 & c. $76 \mathrm{G}>\mathrm{A}$, p.Gly26Ser & 18 & 10 & 8 & 0.027 & 0.074 \\
\hline & $\ln 3$ & rs36204272 & c. $337-18 G>C$ & 7 & 3 & 4 & 0.011 & 0.037 \\
\hline & Ex 3 & & c. $105 A>G$, p.Lys35Lys & 1 & 0 & 1 & 0.002 & - \\
\hline & Ex 4 & rs28933981 & c. $416 \mathrm{C}>\mathrm{T}$, p.Thr139Met & 2 & 0 & 2 & 0.003 & 0.003 \\
\hline & 3'UTR & rs62093482 & c. ${ }^{*} 261 \mathrm{C}>\mathrm{T}$ & 6 & 3 & 3 & 0.009 & 0.028 \\
\hline & 3'UTR & rs1053907197 & c. ${ }^{*} 75 \mathrm{~A}>\mathrm{C}$ & 1 & 1 & 0 & 0.002 & - \\
\hline & 3' near gene & rs 1791228 & c. ${ }^{*} 402 \mathrm{C}>\mathrm{T}$ & 127 & 87 & 40 & 0.194 & 0.463 \\
\hline
\end{tabular}

$\mathrm{AO}$, age-at-onset. Novel variants are highlighted in bold 
. Table 2: Statistically significant results for rare variants associated with $\mathrm{AO}$ variation, using GEE analysis, after Bonferroni correction.

\begin{tabular}{|c|c|c|c|c|c|}
\hline $\begin{array}{c}\text { TTR } \\
\text { Region }\end{array}$ & Rare variant & Genotypes & B & $95 \% \mathrm{Cl}$ & $\begin{array}{c}\mathrm{P}- \\
\text { value }\end{array}$ \\
\hline \multirow{17}{*}{ Promoter } & Intercept & - & 40.1 & {$[36.7 ; 43.6]$} & $<0.001$ \\
\hline & \multirow{2}{*}{ c. $-1993 \mathrm{G}>\mathrm{T}$} & GG (Ref) & - & - & - \\
\hline & & GT & -6.1 & {$[-9.6 ;-2.7]$} & $<0.001$ \\
\hline & \multirow{2}{*}{ rs13381522 } & CC (Ref) & - & - & - \\
\hline & & CT & 21.4 & {$[8.5 ; 34.3]$} & 0.001 \\
\hline & \multirow{2}{*}{ rs546878244 } & GG (Ref) & - & - & - \\
\hline & & GA & -8.1 & {$[-11.6 ;-4.7]$} & $<0.001$ \\
\hline & \multirow{2}{*}{ rs3764477 } & GG (Ref) & - & - & - \\
\hline & & GA & -15.5 & {$[-18.2 ;-12.9]$} & $<0.001$ \\
\hline & \multirow{2}{*}{ rs540872876 } & GG (Ref) & - & - & - \\
\hline & & GA & 18.4 & {$[14.8 ; 21.9]$} & $<0.001$ \\
\hline & \multirow{2}{*}{ rs58616646 } & CC (Ref) & - & - & - \\
\hline & & CT & -18.4 & {$[-21.9 ;-14.8]$} & $<0.001$ \\
\hline & \multirow{2}{*}{ rs146750662 } & CC (Ref) & - & - & - \\
\hline & & CT & -8.1 & {$[-11.6 ;-4.7]$} & $<0.001$ \\
\hline & \multirow{2}{*}{ rs996949357 } & TT (Ref) & - & - & - \\
\hline & & $\mathrm{TC}$ & 11.9 & {$[8.4 ; 15.3]$} & $<0.001$ \\
\hline \multirow{7}{*}{$\begin{array}{l}\text { Coding } \\
\text { and } \\
\text { flanking } \\
\text { regions }\end{array}$} & Intercept & - & 42.0 & {$[39.5 ; 44.5]$} & $<0.001$ \\
\hline & \multirow{2}{*}{$\begin{array}{c}\text { c. } 200+107 \\
T>C\end{array}$} & TT (Ref) & - & - & - \\
\hline & & $\mathrm{TC}$ & 8.19 & {$[5.3 ; 11.1]$} & $<0.001$ \\
\hline & \multirow{2}{*}{ rs28933981 } & CC (Ref) & - & - & - \\
\hline & & $\mathrm{CT}$ & 21.59 & [17.3; 25.9] & $<0.001$ \\
\hline & \multirow{2}{*}{ rs1053907197 } & AA (Ref) & - & - & - \\
\hline & & $A C$ & -7.81 & {$[-10.7 ;-4.9]$} & $<0.001$ \\
\hline
\end{tabular}

Ref, Reference genotype; B, unstandardized coefficient (estimated quantitative effect of each genotype on mean $\mathrm{AO}$ variation according to the intercept, compared with the reference genotype); $\mathrm{Cl}$, confidence interval; significance level set to 0.0125 
Table 3: Haplotype association analysis of all variants identified in TTR locus on ValzoMet TTR carriers.

\begin{tabular}{cccccc} 
Haplotype & $\begin{array}{c}\text { Haplotype } \\
\text { Frequency }\end{array}$ & \multicolumn{2}{c}{ Haplotype Frequency } & $\begin{array}{c}\text { P- } \\
\text { value }\end{array}$ & $\begin{array}{c}\text { P- } \\
\text { permuted* }\end{array}$ \\
\cline { 3 - 4 } ACGCAGGCCAGTGGCCC & 0.664 & 0.706 & 0.625 & 0.1829 & 0.6341 \\
\hline GCGAAGGCCTGTGGCCT & 0.071 & 0.053 & 0.087 & 0.3089 & 0.9956 \\
\hline GCTAAGGCCTGTGGCCT & 0.036 & 0.059 & 0.016 & 0.0721 & 0.2231 \\
\hline GCTAAGGCCTGTGGCCC & 0.034 & 0.036 & 0.032 & 0.8428 & 10.000 \\
\hline GCGAGGGCCTGTGGCCT & 0.033 & 0.035 & 0.032 & 0.8956 & 10.000 \\
\hline GCGAAGGCCTGTGGCCC & 0.026 & 0.018 & 0.033 & 0.4819 & 10.000 \\
\hline ACGCAGGCCAGTGGCCT & 0.019 & 0.021 & 0.017 & 0.8564 & 10.000 \\
\hline GCGAGGGCCTGTGGCCC & 0.017 & 0.018 & 0.016 & 0.9188 & 10.000 \\
\hline ATGCAAGTCAGTGCCCC & 0.017 & 0.000 & 0.032 & 0.055 & 0.1582 \\
\hline ACGCAGGCCAGTGGCTC & 0.012 & 0.009 & 0.016 & 0.6208 & 10.000 \\
\hline ACGCAGGCCAGTAGCCT & 0.012 & 0.017 & 0.008 & 0.5058 & 10.000 \\
\hline * Permutation for 10,00o times. & & & & &
\end{tabular}


Table 4: Predicted transcription factors (TFs) which have lost (disrupted) or gain (new) affinity to bind in the presence of rs3764479/rs3764478variants.

\begin{tabular}{|c|c|c|c|}
\hline Disrupted TF & & New TF & \\
\hline NR1H4 & JUNB & REL & RREB1 \\
\hline MAFG & JUND & NFATC2 & CTCF \\
\hline ATF4 & EGR1 & $A R$ & HSF1 \\
\hline IRF1 & RELA & TCF7L2 & HLF \\
\hline NFIL3 & KLF9 & MXI1 & CEBPG \\
\hline MLX & HMBOX1 & ZBTB7A & RARA \\
\hline ATF7 & NRF1 & MYC & HNF4G \\
\hline NFYA & KLF4 & ESRRA & TEAD2 \\
\hline MEF2B & HEY2 & ZВТВ7B & ZBTB33 \\
\hline TGIF2 & NR4A2 & JUN & GMEB2 \\
\hline GLIS3 & THAP1 & USF2 & NR3C1 \\
\hline NKX3-1 & ZNF263 & BHLHE40 & RORA \\
\hline SOX9 & ETV6 & SREBF1 & MAFF \\
\hline POU6F1 & RELB & NR2C2 & TFCP2 \\
\hline MSC & TFDP1 & $\mathrm{ZIC1}$ & GABPA \\
\hline HSF2 & NFKB2 & TFAP4 & GATA6 \\
\hline FOXP1 & SP1 & MNT & MTF1 \\
\hline \multirow[t]{8}{*}{ ESR1 } & SP3 & ELK3 & HESX1 \\
\hline & TEAD1 & TFEB & RUNX1 \\
\hline & ZNF740 & CLOCK & \\
\hline & TBX15 & NFATC3 & \\
\hline & SPI1 & RUNX3 & \\
\hline & RBPJ & CEBPD & \\
\hline & ELK4 & SREBF2 & \\
\hline & HEY1 & HIF1A & \\
\hline
\end{tabular}


Table 5: Prediction of splice sites and splicing enhancers (ESE) alterations by HSF and the ESEfinder software.

\begin{tabular}{|c|c|c|c|c|c|}
\hline Variant & Splice sites & $\begin{array}{c}\text { Wild-type } \\
\text { CV }\end{array}$ & Val30Met CV & $\begin{array}{c}\text { CV } \\
\text { variation }\end{array}$ & $\begin{array}{c}\text { Distance from } \\
\text { variant }\end{array}$ \\
\hline rs1800458 & acceptor site & 0 & 77 & 77 & 2 nt downstream \\
\hline rs36204272 & donor site & 68.6 & 0 & -68.6 & - \\
\hline rs1791228 & branch point & 60.2 & 85 & 24.8 & 2 nt downstream \\
\hline Variant & SR protein & $\begin{array}{l}\text { Wild-type } \\
\text { MS }\end{array}$ & $\begin{array}{l}\text { Val30Met } \\
\text { MS }\end{array}$ & $\begin{array}{c}\text { MS } \\
\text { variation }\end{array}$ & $\begin{array}{l}\text { Distance from } \\
\text { variant }\end{array}$ \\
\hline \multirow{6}{*}{ rs1800458 } & SC35 & 0 & 2.73 & 2.73 & 6 nt upstream \\
\hline & SC35 & 0 & 2.67 & 2.67 & 2 nt upstream \\
\hline & SC35 & 3.02 & 2.57 & -0.45 & 7 nt upstream \\
\hline & SF2/ASF site & 0 & 1.99 & 1.99 & 6 nt upstream \\
\hline & SF2/ASF site & 4.64 & 2.73 & -1.91 & 4 nt upstream \\
\hline & $\begin{array}{l}\text { SF2/ASF (IgM- } \\
\text { BRCA1) site }\end{array}$ & 4.56 & 2.86 & -1.70 & 4 nt upstream \\
\hline \multirow{4}{*}{ rs28933981 } & SRp40 & 2.81 & 0 & -2.81 & 6 nt upstream \\
\hline & SRp55 & 3.89 & 0 & -3.89 & 2 nt upstream \\
\hline & SF2/ASF site & 2.03 & 0 & -2.03 & - \\
\hline & $\begin{array}{l}\text { SF2/ASF (IgM- } \\
\text { BRCA1) site }\end{array}$ & 2.87 & 0 & -2.87 & - \\
\hline rs62093482 & SC35 & 3.73 & 3.5 & -0.23 & 3 nt upstream \\
\hline \multirow[b]{2}{*}{ rs1791228 } & SRp40 & 3.05 & 3.71 & 0.66 & 3 nt upstream \\
\hline & $\begin{array}{l}\text { SF2/ASF (IgM- } \\
\text { BRCA1) site }\end{array}$ & 2.33 & 2.58 & 0.25 & 1 nt upstream \\
\hline
\end{tabular}

CV, Consensus value; MS, Matrix score; nt, nucleotides; SR protein: Serine and arginine-rich (SR) proteins; $\mathrm{SC}_{35}$, Serine/arginine-rich splicing factor; SF2/ASF, pre-mRNA-splicing factor SF2/alternative splicing factor; SRp40, Splicing factor, arginine/serine rich 40 kDa; Srp55, Splicing factor, arginine/serine rich $55 \mathrm{kDa}$. 